\title{
Atlas of Ultrasound-Guided Regional Anesthesia, 3rd Edition
}

\author{
Andrew T. Gray. Elsevier, Philadelphia, 2019, \$222.72, 422 pages, ISBN 978-0-323-50951-0
}

\author{
Sathish Shanmuganathan, MD • Derek Dillane, MD $\mathbb{D}$
}

Received: 14 August 2018/Accepted: 14 August 2018/Published online: 29 August 2018

(C) Canadian Anesthesiologists' Society 2018

The widespread availability of instructional resources for ultrasound-guided regional anesthesia (UGRA), from video-sharing websites to comprehensive workshops, has resulted in an educational market that is relatively unsaturated by textbooks and atlases. The revised and updated third edition of Atlas of Ultrasound-Guided Regional Anesthesia will satisfy UGRA devotees seeking a less ephemeral reference. Owners of the previous, second edition will notice the addition of 12 new chapters detailing recently described techniques, the preponderance to be found in the sections on the trunk and head and neck. In a departure from previous editions, 14 of the 75 chapters are by contributing authors, many being "household names" in regional anesthesia. Despite its multi-author nature, there is a uniformity to the structure of the book, and the editor is to be commended for the seamless fusion of what are presumably disparate writing styles.

The textbook's 75 chapters are organized into seven sections. The introductory section addresses the physics of ultrasound, artifacts, and scanning and needling approaches. Readers may be struck by the succinctness of this section's chapters - indeed, some are only four sentences long. However, the information imparted is germane and sufficient for a fundamental understanding of the ensuing sections. The often neglected yet crucial topic of end points in UGRA is covered in a chapter titled Sonographic Signs of Successful Injections. This chapter notes the unlikelihood of an intervening fascial plane serving as a barrier to local anesthetic diffusion if more than half the nerve border is encompassed by local anesthetic. This observation - empirical if not entirely evidence-based - is emblematic of the wealth of practical advice on display. One of the editorial traits of the book is the dissemination of new information throughout the

S. Shanmuganathan, MD · D. Dillane, MD $(\varangle)$

University of Alberta, Edmonton, AB, Canada

e-mail: ddillane@shaw.ca entirety of each chapter. Readers who pay close attention to figure legends will be rewarded for the attention to detail e.g. legends for the ultrasound images in the chapter on the sonographic appearance of muscle note how the direction in which the muscle fibres run can be used to distinguish between pectoralis major and minor or between the internus oblique and transversus abdominis muscles.

Most of the Atlas is dedicated to outlining techniques for blockade of the upper and lower extremities, trunk, and head and neck. The sections on upper and lower limb blocks, replete with nuanced anatomic detail and irregularities, were undoubtedly written by an expert who performs these techniques on a daily basis and has given significant thought to his craft. High-resolution sonograms are plentiful though the novice sonographer may be frustrated by the sparse labelling on some images. Clinical applications for each block, although occasionally present in the introduction to each chapter, are not explicitly imparted in a consistent manner. Summarizing the clinical applications would have been a useful addition to the Key Points box insets, which are present in approximately half of the chapters devoted to block techniques. At a glance, these boxes codify key anatomical findings, block ergonomics, transducer and needle choice, and the specific approaches to each block. This feature has been omitted from occasional chapters regarding some of the more popular blocks-e.g., blocks above the clavicle, which may preclude its use as a bedside guide for novices of these particular techniques.

The chapters on sciatic nerve blockade are particularly informative and provide some fascinating insights, such as why the tibial nerve is more clearly visible than the peroneal nerve in the popliteal fossa (i.e., it is not only about size) or why a bayonet artifact is not uncommon at this location. The section that addresses the trunk contains new chapters on pectoralis, transversus thoracis muscular plane, quadratus lumborum, pudendal, and neuraxial blockade. Another addition is an extensive chapter on paravertebral blockade. Indeed, in this longest chapter by a 
considerable measure, the text is cogent and the accompanying illustrations, clinical photographs, and sonograms provide ample information to satisfy even the most discerning reader. Those looking for an all-in-one resource may be concerned by the sparing use throughout the book as a whole, of anatomical line drawings. Considering the panoply of included ultrasound images, this lack may have been a conscious effort to limit the size and weight of the end product. The illustrations that have been included are, for the most part, reproduced from wellknown anatomical texts and of the highest quality.

The final section, Safety Issues, contains two oddly complementary chapters. The first is an authoritative, evidence-based discussion of complications which may occur during UGRA. In it, the authors focus on practical suggestions for the prevention of these complications, rather than providing guidelines for their management should they occur. The final chapter, ostensibly beyond the remit of a regional anesthesia atlas, is a unique and thought-provoking overview of the challenges associated with practicing regional anesthesia in resource-constrained environments.
In addition to the usual print version, the Atlas of Ultrasound-Guided Regional Anesthesia, $3^{\text {rd }}$ Edition has been fully reproduced in an electronic version with an excellent, searchable index. It is available in desktop, tablet, or smartphone formats that provide access to 20 video clips covering many of the block techniques discussed in the book. The e-book can be downloaded for convenient offline use.

In summary, Atlas of Ultrasound-Guided Regional Anesthesia, $3^{\text {rd }}$ Edition is an expertly written text enriched by an abundance of high-quality ultrasound images. However, it is not a recipe book, and synthesis of the provided information requires an effort from the reader. For the intermediate-to-advanced practitioner of UGRA, this book is an excellent resource and a worthy addition to the bibliography in this field.

Conflicts of interest None declared.

Editorial responsibility This submission was handled by Dr. Hilary P. Grocott, Editor-in-Chief, Canadian Journal of Anesthesia. 